\title{
Sustained T cell Rap1 activation protects against Experimental Autoimmune Encephalomyelitis (EAE) via modulation of $\mathrm{T}$ cell responses
}

\author{
G Franco Salinas, S Krausz, W Dontje, P P Tak, D Baeten, K Reedquist \\ From 5th European Workshop on Immune-Mediated Inflammatory Diseases \\ Sitges-Barcelona, Spain. 1-3 December 2010
}

\section{Introduction}

Rap1 is signalling molecule that modulates $\mathrm{T}$ lymphocyte trafficking and activation upon antigen stimulation. We demonstrated that transgenic mice with $\mathrm{T}$ cells expressing constitutively active Rap1 (RapV12) are protected from experimental arthritis. Here, we aimed to identify the mechanisms of protection by using a TCR transgenic model of MOG-induced EAE (2D2 mice).

\section{Methods}

Flow cytometry and ELISA were used to analyse the phenotype and cytokine profile of MOG-specific autoreactive $\mathrm{T}$ cells in 2D2 xRapV12, 2D2, RapV12 and wild type (WT) mice, either in basal conditions or after in vivo priming with MOG peptide. Clinical EAE was monitored for 30 days.

\section{Results}

Under homeostatic conditions, there was a strong reduction in the number of autoreactive $\mathrm{T}$ cells in 2D2xRapV12 versus $2 \mathrm{D} 2$ animals in both the naïve and memory compartments, indicating that constitutive Rap1 activation enforces central tolerance mechanisms. Analysis of the remaining autoreactive $\mathrm{T}$ cells showed no differences in $\mathrm{T}$ cell subsets, proliferation, apoptosis, expression of costimulatory molecules and production of pro-inflammatory cytokines. After in vivo priming with MOG, however, we observed a profound inhibition of TNF production by $\mathrm{T}$ cells expressing RapV12 whereas there was a slight increase in IFNgamma and IL-17 production. To evaluate the pathophysiological relevance of these alterations of autoreactive T cells, EAE was induced in 2D2xRapV12, 2D2, RapV12 and WT littermates. In the 2D2 model, where a significant number of autoreactive $T$ cells are still present despite the constitutive RapV12, we observed no difference in EAE scores but an improved survival in the $2 \mathrm{D} 2 \mathrm{xRapV} 12$ versus $2 \mathrm{D} 2$ mice $(\mathrm{p}=0.04)$. In WT mice, sustained Rap1 activation led to increased survival $(\mathrm{p}=0.02)$ as well as lower EAE scores $(\mathrm{p}=0.07)$.

\section{Conclusion}

Sustained activation of Rap1 reduces the autoreactive $\mathrm{T}$ cell pool and affects pro-inflammatory cytokine production by the remaining autoreactive $\mathrm{T}$ lymphocytes. As in experimental arthritis, these quantitative and qualitative effects are associated with a protection from autoimmune disease in EAE.

Published: 25 November 2010

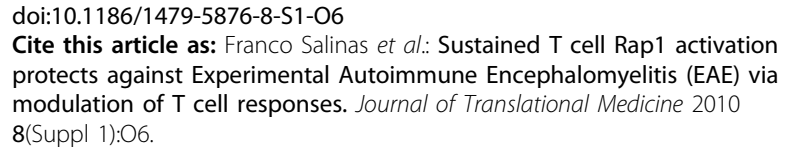

Cite this article as: Franco Salinas et al: Sustained T cell Rap1 activation protects against Experimental Autoimmune Encephalomyelitis (EAE) via modulation of T cell responses. Journal of Translational Medicine 2010 8(Suppl 1):O6. 